\title{
Improving the reliability of visual acuity measures in young children
}

\author{
Paul V. McGraw, ${ }^{1}$ Barry Winn, ${ }^{1}$ Lyle S. Gray ${ }^{2}$ and David B. Elliott ${ }^{1}$ \\ ${ }^{1}$ Department of Optometry, University of Bradford, Richmond Road, Bradford BD7 1DP, West \\ Yorkshire, UK and ${ }^{2}$ Department of Vision Sciences, Glasgow Caledonian University, \\ Cowcaddens Road, Glasgow G4 0BA, UK
}

\begin{abstract}
Summary
Whilst the methodology of adult letter acuity measurement has been substantially refined over the last two decades, relatively little development has occurred in methods for quantifying letter acuity in young children. This study compares a recently developed visual acuity test (Glasgow Acuity Cards), which incorporates several key design features used in adult test charts to improve the sensitivity and reliability of visual acuity measurements. The equivalence of acuity measurements made with Glasgow Acuity Cards were compared with the Bailey-Lovie logMAR chart and Snellen chart in adults, and with traditional Single Letter Acuity and a modified Single Letter Acuity test in children. The test-retest reliability of acuity measurements made with Glasgow Acuity Cards and the Single Letter Acuity tests were also assessed in a large group of visually normal children. In addition, the ability of the pre-school letter acuity tests to detect differences in acuity between the two eyes, and to detect amblyopia were examined. Ninety-five percent of vision measurements made with the Bailey-Lovie chart and Glasgow Acuity Cards differ by less than 0.07 log unit. Furthermore, the sensitivity of Glasgow Acuity Cards to detecting changes in acuity longitudinally and inter-ocular differences in acuity is considerably greater as compared with traditional Single Letter Acuity tests. Improvements in paediatric acuity chart design are important for the effective detection and management of children with amblyopia. (C) 2000 The College of Optometrists. Published by Elsevier Science Ltd.
\end{abstract}

\section{Introduction}

Visual acuity testing using Snellen-based letter charts remains the principal measure of functional visual integrity used by eye care professionals, despite the well documented limitations of these charts (Gibson and Sanderson, 1980; Wick and Schor, 1984; LovieKitchin, 1988). Detection of change in visual acuity or

Received: 28 January 1999

Revised form: 5 August 1999

Correspondence and reprints requests to: Paul V. McGraw.

E-mail address: p.v.mcgraw@bradford.ac.uk (Paul V. McGraw). deviation from normality is important clinically as it is likely to influence the future course of treatment and management of a particular condition (Bailey et al., 1991). When visual acuity or any other continuous variable is measured or scored there exists a statistical probability that values will differ despite the fact that no real change has occurred (Bailey et al., 1991). Conversely, real changes can occur which are not detected as the score remains unaltered (Bailey et al., 1991). Therefore, to decide whether a real change has occurred, the clinician needs to establish confidence limits for the test. While these limits depend on the inherent variability within a test, recent reports (Bailey et al., 1991; Reeves et al., 1987) have shown that the 
selection of appropriate grading scales can improve clinical measurement and allow differences in scores to be determined with increased confidence.

Test charts for the measurement of visual acuity in adults (Bailey and Lovie, 1976; Ferris et al., 1982; Regan, 1988) have improved both the accuracy and reliability of letter acuity measures (Lovie-Kitchin, 1988) by introducing novel design features and scoring systems. The Bailey-Lovie chart and the Early Treatment of Diabetic Retinopathy Study (ETDRS) chart have become 'gold standard' measures of visual acuity in clinical research and clinical trials (Ferris and Bailey, 1996). However, these important developments in test chart design have only recently been paralleled in the design of tests used to measure specifically the visual acuity of young children (McGraw and Winn, 1993).

The accurate measurement of letter acuity in children is particularly important if early detection of developmental anomalies such as amblyopia is to be achieved. Amblyopia, with a prevalence of approximately $2-3 \%$, remains the major cause of visual dysfunction in the first two decades of life (von Noorden, 1985) and is defined clinically as reduced visual acuity in an otherwise healthy and optically corrected eye. Although reduced acuity can be demonstrated in very young children and infants using behavioural (Teller, 1979) and electrophysiological (Sokol, 1978) techniques, the clinical determination of letter acuity usually commences when the child is old enough to cooperate and interact with the examiner. By the age of 3.5 years, approximately $80 \%$ of children will co-operate with letter acuity testing (Egan and Brown, 1984).

Letter acuity is habitually used to monitor longitudinally the effectiveness of amblyopia therapy, therefore a method of measurement must be devised which is both accurate and repeatable. Since the majority of children referred for orthoptic assessment in the UK are aged 3-5 years (Kendall et al., 1989), there is a need for a test of letter acuity incorporating design features which have been shown to improve letter chart performance in adults (Lovie-Kitchin, 1988).

Single letter acuity measures such as the SheridanGardiner test (Sheridan, 1963) and Sight Test for Young Children and Retardates (STYCAR) (Sheridan, 1963) remain the primary measure of visual acuity in pre-school children (Stewart-Brown et al., 1988). The drawbacks of the use of single letter acuity measures in children with amblyopia are well documented (Hilton and Stanley, 1972; Youngson, 1975; Fern et al., 1986). This has led to the development of alternative tests (Atkinson et al., 1985, 1988; Salt et al., 1995) in an attempt to improve the clinician's ability to detect amblyopia in pre-school children. However, many tests designed to achieve this aim employ a similar scoring system and progression of letter sizes to the traditional single letter acuity tests. Unfortunately, it is precisely these features that determine the utility of the test in detecting changes in acuity (Bailey et al., 1991). Therefore, although they may improve the detection of amblyopia in pre-school children, their ability to describe changes in acuity and differences in acuity between the two eyes are likely to be comparable with Snellen-based single letter acuity tests.

A new visual acuity test (Glasgow Acuity Cards, McGraw and Winn, 1993) has recently been developed which incorporates several key design features used in adult test charts (Bailey and Lovie, 1976) to improve the sensitivity and reliability of visual acuity measurements in young children, and introduces features of particular value in the measurement of visual acuity in amblyopic eyes. In this study the effects of incorporating such features into letter acuity tests for young children are examined.

The present study investigates three main areas:

\section{Adults: comparison between Glasgow Acuity Cards, Baile-Lovie chart and Snellen chart}

The equivalence of acuity measurements made with Glasgow Acuity Cards was compared with the gold standard' Bailey-Lovie logMAR chart in adults. For comparative purposes Glasgow Acuity Cards were also compared with the traditional Snellen chart.

2. Visually normal children: comparison between Glasgow Acuity Cards, Single Letter Acuity and Single Letter Acuity with a Log scale and their test-retest reliability

The equivalence of acuity measurements made with Glasgow Acuity Cards were compared with traditional Single Letter Acuity and a modified version of a Single Letter Acuity test, which utilises a logarithmic letter size progression. A comparison with Single Letter Acuity has been chosen since this is the most commonly used method used for measurement of visual acuity in pre-school children (Stewart-Brown et al., 1988).

The test-retest reliability of acuity measurements made with Glasgow Acuity Cards and a Single Letter Acuity test were assessed in a large group of visually normal children. In addition, to allow the effects of both scoring system and letter size progression to be evaluated, a comparison with the modified Single Letter Acuity ( $\mathrm{Log})$ test was included. These data provide $95 \%$ confidence limits for a significant change in letter acuity, and are therefore of particular relevance to children undergoing treatment such as amblyopia therapy where an improvement in letter acuity is sought. 


\section{Study of inter-ocular acuity differences in visually} normal children and children with amblyopia

To provide a comparison between the ability of Glasgow Acuity Cards, Single Letter Acuity and Single Letter Acuity (Log) at detecting differences in acuity between the two eyes. Confidence limits for inter-ocular acuity differences are established in an additional group of visually normal children. These data were then used to assess the sensitivity of each test in detecting unilateral amblyopia.

\section{Experiment 1. Adults: comparison between Glasgow Acuity Cards, Bailey-Lovie chart and Snellen chart}

\section{Methods}

The design features of Glasgow Acuity Cards have been described in detail elsewhere (McGraw and Winn, 1993), however a brief summary of the principal features of the chart are presented below.

Glasgow Acuity Cards are viewed directly at a distance of $3 \mathrm{~m}$, since it has been suggested that some children may perform slightly better at this reduced distance for psychological reasons (Kitao, 1960; Sheridan, 1974; Brant and Nowotny, 1976). Furthermore, the $3 \mathrm{~m}$ test distance reduces test times and increases patient co-operation particularly in poorly motivated children (Sheridan, 1960; Smorvik and Bosnes, 1976; Pickert and Wachs, 1980). A regular geometric progression of letter sizes is employed whose ratio is equal to ${ }^{10} \sqrt{ } 10(0.1 \log$ unit). This letter size progression has been used effectively in the Bailey-Lovie chart (Bailey and Lovie, 1976), and provides approximately equal increments in resolution across the chart (Dreyer, 1964; Westheimer, 1979). Six letters (X V O H U Y) of approximately equal legibity (Bennett, 1965) are used. Since children develop horizontal laterality later than vertical laterality (Graham et al., 1960; Wohlwill, 1960; Rudel and Teuber, 1963), all letters used are symmetrical about the vertical mid-line to avoid confusion when used with younger children. Four letters appear on each line to ensure a constant visual demand (Bailey and Lovie, 1976) at each acuity level. Measured visual acuities range from $3 / 19$ to $3 / 1.5$ (equivalent to $6 / 38-6 / 3$ ) at $3 \mathrm{~m}$, allowing parametric statistical analysis of the complete distribution of acuity scores (Wild and Hussey, 1985).

Clearly, it is unlikely that young children will be able or willing to read many rows of letters to allow a measure of threshold visual acuity. To overcome this problem, yet still obtain an accurate score, a series of screening cards are used to identify a starting point for the measurement of line acuity.

Surrounding contours have been incorporated into the test to improve sensitivity in amblyopia detection. The letter spacing is equal to 0.5 letter diameter to increase the amount of contour interaction (Flom et al., 1963). Standardisation of the crowding effect at each acuity level is controlled by appropriate interletter spacing and the use of a crowding bar which surrounds the four letter array.

A simple scoring system has been devised which is based on the log of the minimum angle of resolution. The modified scoring system designates $6 / 6$ a score of 1 and $6 / 60$ a score of 0 with visual acuities less than $6 /$ 60 carrying a negative sign. As there are four letters per line and the difference between lines is $0.1 \mathrm{log}$ unit each individual letter correctly identified scores 0.025 $\log$ unit. The endpoint is reached when the child cannot identify any letters on a particular line. This log-based method of scoring offers several advantages. Specifically, this scoring-by-letter technique effectively makes the clinical grading scale four times finer than a scoring-by-row technique (Bailey et al., 1991), therefore enhancing the sensitivity to detecting changes in acuity.

Memorisation and inter-session variability are an important consideration (Ferris et al., 1982; Elliott and Sheridan, 1988), particularly when letter acuity is used to assess the efficacy of amblyopia therapy or any other treatment modality. Training effects are minimised by having multiple versions of the acuity test. Different charts can be used if frequent re-testing of acuity is indicated or if an acuity score requires verification on the same visit.

Testing protocol - Glasgow Acuity Cards (GAC). The test was viewed at $3 \mathrm{~m}$ and was externally illuminated to $150 \mathrm{cdm}^{-2}$. The initial letter acuity level was determined using the screening cards. The subject was encouraged to respond to each letter in the series, which correspond to the linear acuity levels within the chart, until an error was made. The last successful response was used to determine the starting point for the measurement of line acuity. The appropriate card was then selected and the subject attempted to identify each of the four letters presented. If the patient was able to identify correctly two or more letters on a line then the next card in the series was presented.

Testing protocol - Snellen Chart (SC). A standard Snellen chart was used, which was viewed at a distance of $6 \mathrm{~m}$ and externally illuminated to $150 \mathrm{cdm}^{-2}$. The acuity scale of the chart used ranged from $6 / 60$ to 6/4.5. A normal clinical procedure was adopted for scoring the Snellen chart, vision being defined as the smallest line at which a majority of letters were read.

Testing protocol - Bailey-Lovie Chart (BLC). The chart was viewed at a distance of $6 \mathrm{~m}$, and was 
a

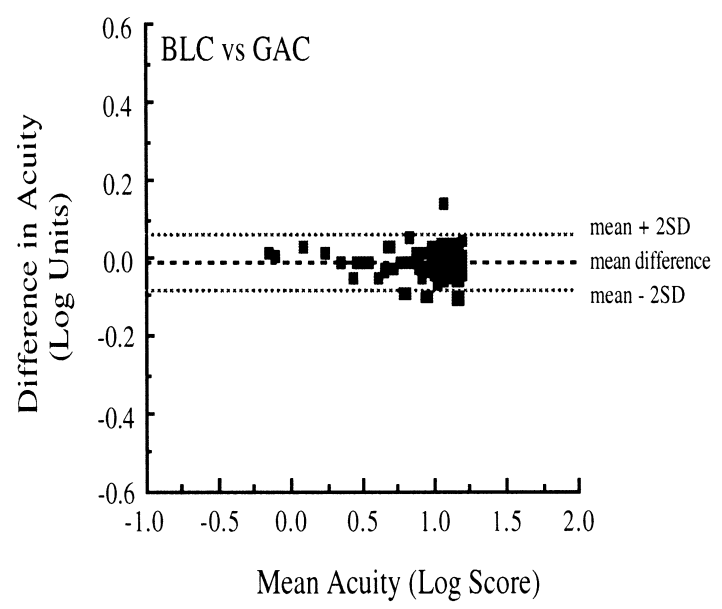

b

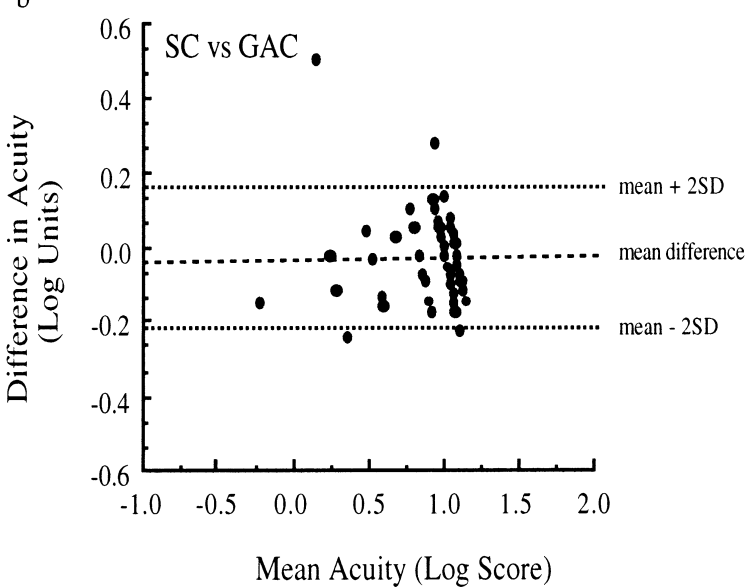

c

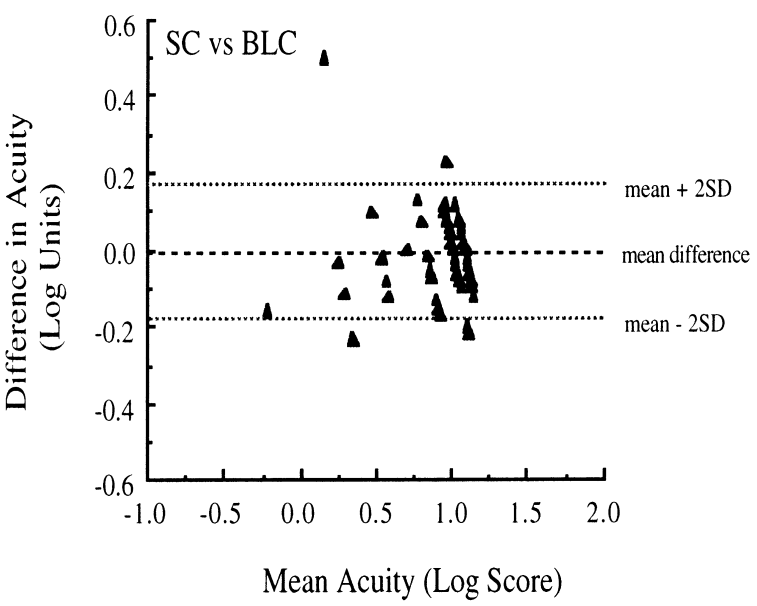

Figure 1. Scatterplots of the difference in acuity against the mean acuity for (a): Glasgow Acuity Cards vs Bailey-Lovie chart; (b): Snellen chart vs Glasgow Acuity Cards; (c): Snellen chart vs Bailey-Lovie chart, for 103 adult subjects. In all chart comparisons the acuity score of the second test in the title was subtracted from that of the first (e.g. BLC vs GAC is Bailey Lovie chart score-Glasgow Acuity Cards score). externally illuminated to $150 \mathrm{cdm}^{-2}$. Letter sizes ranged from $6 / 60$ to $6 / 3$ Snellen equivalent. An interpolated scoring systen where each letter on the chart carries a score of $0.02 \log$ units was used. As with the two tests above the subject was encouraged to guess when unsure.

If the subject was unable to identify any letters on the largest line of the Glasgow Acuity Cards or the Bailey-Lovie chart they were moved closer in $0.10 \mathrm{log}$ unit steps until the majority of the largest line could be identified. In the case of the Snellen chart the test distance was reduced to $3 \mathrm{~m}$. Acuity measurements from all three charts were scored using the Glasgow Acuity Cards notation which is defined as: GAC Scor$\mathrm{e}=(1-\log \mathrm{MAR})$

\section{Subjects}

Unaided distance vision was measured in one eye of 103 adult subjects (median age $=21.5$ years, range $=50$ years) using Glasgow Acuity Cards, the Bailey-Lovie chart and the Snellen chart. The order of presentation of each of the tests was randomised. All subjects were screened prior to testing to ensure the absence of ocular disease or any other non-refractive causes of reduced vision. Written consent was obtained from all subjects prior to experimental testing.

\section{Method of data analysis}

Comparison of acuity scores obtained for the adult and child test charts were assessed in terms of the standard deviation of the distribution of differences in score between tests (Bland and Altman, 1986). This method allows the assessment of agreement in method comparison paradigms. For comparative purposes Pearson product moment correlation coefficients and Spearman rank correlation coefficients (where appropriate) have also been calculated.

\section{Results and discussion}

Scatterplots of the difference in acuity score against the mean acuity score for each set of test comparisons are shown in Figure $1 a-c$. The results indicate a mean difference in acuity score between the Bailey-Lovie chart and Glasgow Acuity Cards of $-0.013 \log$ unit with the limits of agreement being $\pm 0.067 \log$ unit. Therefore $95 \%$ of vision measurements made with the Bailey-Lovie chart and Glasgow Acuity Cards will differ by less than $0.07 \log$ unit (i.e. less than three letters on GAC). Linear regression analysis revealed a highly significant correlation $\left(r^{2}=0.98\right)$ for this test comparison $(p<0.0001)$.

The limits of agreement between the Bailey-Lovie chart and Glasgow Acuity Cards are considerably narrower than the $95 \%$ ranges for either of these charts 
when compared to the Snellen chart (approximately two lines). Significant correlations were found for all three sets of test comparisons (see Table 1).

LogMAR-based tests have become the accepted 'gold standard' measurement of adult letter acuity in research studies (Ferris and Bailey, 1996). Letter acuity tests used clinically for monitoring change should be able to offer similar levels of performance. Although Glasgow Acuity Cards were designed principally to allow determination of letter acuity in pre-school children it is essential that the performance of this new test is comparable to the Bailey-Lovie chart as both tests employ similar features. The results show that Glasgow Acuity Cards provide an equivalent measure of letter acuity in adults when compared to the Bailey-Lovie chart.

The broader confidence limits found with Snellen chart comparisons may be explained by a number of factors. Firstly, subjects with a good level of vision are constrained by the truncation of the measuring scale on the Snellen chart as many young adults have a visual acuity better than 6/4.5 (Elliott et al., 1995); secondly, inter-letter spacing and the presence of flanking contours vary across the acuity range. On the smallest line, letter spacing is greater than one letter width, thus reducing the deleterious effect of contour interaction (Flom et al., 1963) compared with levels produced by both Glasgow Acuity Cards and the BaileyLovie chart. At the largest letter size on the Snellen chart, subjects are presented with an unflanked recognition task, which may give an artificially increased vision score (Flom et al., 1963).

\section{Experiment 2. Visually normal children: comparison between Glasgow Acuity Cards, Single Letter Acuity and Single Letter Acuity with a Log scale and their test-retest reliability}

\section{Methods}

In addition to Glasgow Acuity Cards two other test charts were used in this section. These are described below.

Single Letter Acuity (SLA). The single letter acuity test used was the Cambridge Singles which are part of the Cambridge Crowding Cards set. This test was administered at a test distance of $3 \mathrm{~m}$, and is similar in format to the Sheridan-Gardiner test. It employs five optotypes $(\mathrm{H}, \mathrm{O}, \mathrm{T}, \mathrm{V}, \mathrm{X})$, different numbers of which are presented at each acuity level. The irregular letter size progression is identical to that of the Cambridge Crowding Cards and other Snellen-based tests. Vision was defined as the smallest acuity level at which a majority of letters were correctly matched.

This chart was included in the study since it has letter sizes which range from $6 / 60$ to $6 / 3$, which avoids good acuity scores being constrained by truncation of the measuring scale. Single letter acuity tests are widely used to assess vision in pre-school children and to monitor the effects of amblyopia therapy such as occlusion therapy. Since many of the letter charts designed for pre-school children adopt the progression of letter sizes used in the Snellen chart and define threshold acuity as the smallest line at which a majority of letters is correctly identified, the sensitivity of tests such as the Cambridge Crowding Cards, in detecting changes in acuity should be similar in value to this single letter acuity test.

Single Letter Acuity - Logarithmic progression of letter sizes $(S L A(L))$. This test employs the same six optotypes as the Glasgow Acuity Cards (X V O H U $\mathrm{Y})$ across the same acuity range (Snellen equivalent of $6 / 38-6 / 3)$ and is viewed at a distance of $3 \mathrm{~m}$. Letter size reduces in a regular geometric fashion, with a ratio of ${ }^{10} \sqrt{ } 10$. Three separate unflanked single letters are presented at each acuity level. Standard clinical procedures are used for scoring with acuity defined as the smallest letter size at which two of the three letters were correctly identified. This test was designed specifically for use in this study, to examine the effect of adopting a log scale on test-retest reliability.

\section{Testing protocol}

Measurements of acuity made with Glasgow Acuity Cards, Single Letter Acuity and Single Letter Acuity (Log) charts, were carried out under standard room illumination (500 lux-measured at chart surface, approximately $151 \mathrm{cdm}^{-2}$ ). Tests were carried out in ran-

Table 1. Mean difference, standard deviation and $95 \%$ ranges for the three adult test comparisons

\begin{tabular}{|c|c|c|c|c|c|}
\hline & Mean difference & Standard deviation & $95 \%$ range & $r^{2} \&$ Rho & $p$ Value \& Alpha \\
\hline Bailey-Lovie Chart vs Glasgow Acuity Cards & -0.013 & 0.034 & \pm 0.067 & 0.981 & 0.0001 \\
\hline Snellen Chart vs Glasgow Acuity Cards & -0.027 & 0.095 & \pm 0.19 & $0.754^{\mathrm{a}}$ & $<0.01 \Delta^{\mathrm{b}}$ \\
\hline Snellen Chart vs Bailey-Lovie Chart & -0.012 & 0.092 & \pm 0.18 & $0.783^{\mathrm{a}}$ & $<0.01 \Delta^{\mathrm{b}}$ \\
\hline
\end{tabular}

${ }^{a}$ Rho values from Spearman rank correlation coefficient.

${ }^{\mathrm{b}}$ Alpha values from Spearman rank correlation coefficient. 
dom order. Retest sessions were carried out under identical conditions. All acuity tests were again scored using the Glasgow Acuity Cards log scoring system (1-logMAR). Subjects read down every acuity chart until they were unable to identify correctly any letters on a particular line, all subjects being encouraged to guess until this point was reached.

\section{Subjects}

Unaided vision was measured in one eye of 116 visually normal children (mean age $=5.32 \pm 1.15$ years) using Glasgow Acuity Cards, Single Letter Acuity test and Single Letter Acuity (Log) test. In addition, retest data were collected from 68 of the children to allow the calculation of the $95 \%$ confidence limits for a detection of change in visual acuity for each test. Clinically, test and retest sessions are often separated by a number of weeks, therefore, for estimates of the significance of change to be relevant to clinical follow-up, an interval of 4 weeks separated test and retest sessions.

\section{Method of data analysis}

The reliability of acuity scores for the Glasgow Acuity Cards and the Single Letter Acuity (log) tests have been assessed by plotting the frequency distribution of the test-retest discrepancies. From direct observation of the plot (Elliott et al., 1991), the acuity range in which at least $95 \%$ of the data lie, can be determined. This is acheived by simply summing the frequency from each discrepancy value (from smallest to largest) until $95 \%$ is reached. This method is particularly useful when tests use a quasi-continuous scale. Due to the irregular progression of letter size used in the Single Letter Acuity test, direct observation of the data is inappropriate. In this case the $95 \%$ confidence limits can be established by multiplying the standard deviation of the discrepancy distribution by 1.96 , this figure is termed the coefficient of repeatability (COR) (Bland and Altman, 1986).

Recently a statistical model has been developed (Bailey et al., 1991) where the expected concordance between test and retest comparisons can be predicted from the ratio of the scale increment to the standard deviation of the test-retest discrepancy distribution. Furthermore, 95\% confidence limits can be predicted from a given ratio of scale increment to standard deviation. The degree of concordance and the width of the 95\% confidence limits predicted by the model are used for comparison with experimental results. The effects of changing the test scoring procedure on the confidence limits, are also evaluated.

\section{Results and discussion}

Scatterplots of the difference in acuity score
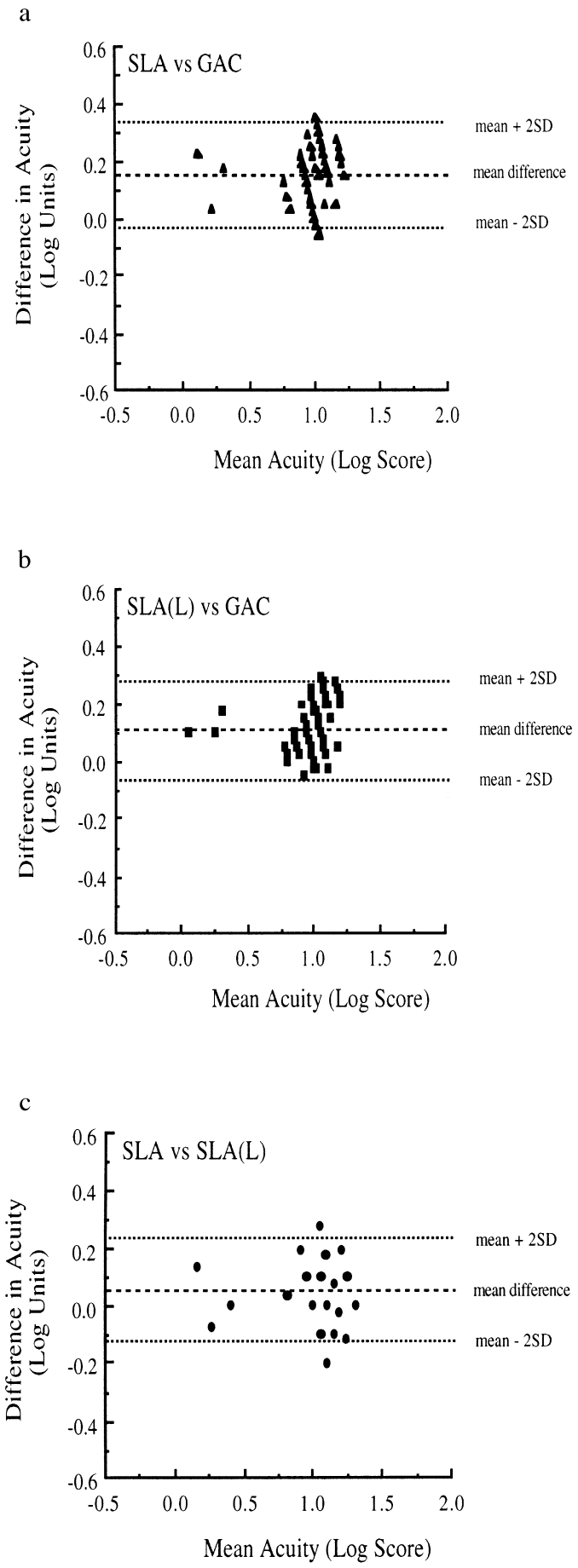

Figure 2. Scatterplots of the difference in acuity against the mean acuity for (a): Single Letter Acuity vs Glasgow Acuity Cards; (b): Single Letter Acuity (Log) vs Glasgow Acuity Cards; (c): Single Letter Acuity vs Single Letter Acuity (Log), for 116 child subjects. In all chart comparisons the acuity score of the second chart in the title was subtracted from that of the first (e.g. SLA vs GAC is Single Letter Acuity score-Glasgow Acuity Cards score). 
against the mean acuity score are shown in Figure $2 a-c$ for the three acuity chart comparisons.

The results of these plots are summarised in Table 2, which gives the mean difference, standard deviation of the differences, $95 \%$ range $( \pm 1.96$ standard deviations), the rho and alpha values from Spearman rank correlation analysis. These plots demonstrate similar $95 \%$ ranges for all three comparisons.

Comparisons of Glasgow Acuity Cards with both Single Letter Acuity and Single Letter Acuity (Log) tests showed a substantial bias in the mean difference of acuity scores. As the Glasgow Acuity Cards score was subtracted from the Single Letter Acuity and Single Letter Acuity ( $\log )$ scores, this indicates that both these tests tended to yield a higher (better) acuity score than Glasgow Acuity Cards. As Glasgow Acuity Cards incorporate features to introduce constant contour interaction, such a difference is expected. The largest mean difference was found for the comparison of Glasgow Acuity Cards with Single Letter Acuity, which was approximately 0.15 $\log$ unit (one and a half lines better with Single Letter Acuity). A difference of approximately 0.10 $\log$ unit (one line better with Single Letter Acuity (Log)) was found between Glasgow Acuity Cards and Single Letter Acuity (Log). None of the children involved in the study were able to reach the highest point on the Glasgow Acuity Cards acuity scale (1.3 GAC log score or $6 / 3$ Snellen equivalent).

The mean difference in acuity scores between Glasgow Acuity Cards and Single Letter Acuity (Log), may in part be due to the effects of contour interaction. This value is similar to that found for 5-7 year olds in a previous study (0.10 log unit), utilising an identical inter-letter spacing (Atkinson et al., 1988). Fern et al. (1986) published a report incorporating data from a number of studies investigating isolated and crowded acuities. They reported a difference between crowded and isolated acuity of $0.09 \log$ unit in 4 year old children and $0.05 \log$ units in 5 year olds. The likely cause of the discrepancy between the value obtained in this study and that arrived at by Fern et al. (1986) are differences in inter-letter spacing, producing varying degrees of contour interaction. The superior performance achieved by young children when tested with unflanked single optotypes is well documented (Fern and Manny, 1986). However, it is important to note that Glasgow Acuity Cards and Single Letter Acuity (Log) differ not only in the provision of flanking contours but also in their scoring procedure. Glasgow Acuity Cards employ an interpolated scoring system whereas Single Letter Acuity $(\log )$ is scored per line. Therefore, the mean difference of 0.105 log unit between Glasgow Acuity Cards and Single Letter Acuity $(\log )$ may also reflect differences due to the scoring procedure.

Figure $3 a-c$ shows the distribution of differences between test and retest scores for the three child acuity tests. Figure $3 a$ shows a frequency distribution plot of test-retest discrepancies for Glasgow Acuity Cards. It can be seen from this figure that at least $95 \%$ of the test-retest discrepancies did not exceed four letters (0.10 log unit). A clinically significant change must therefore fall outside this boundary and is defined by the next incremental step in the acuity scale. As the increments in the Glasgow Acuity Cards scale are $0.025 \log$ unit, a clinically significant change, or difference in score, becomes $\pm 0.125 \log$ unit. This can be contrasted with Figure $3 b$ which shows a similar plot for Single Letter Acuity ( $\log )$. For this particular test the $95 \%$ confidence intervals are applied to a change in score of $\pm 0.20 \mathrm{log}$ unit. A clinically significant difference must exceed the $\pm 0.20 \log$ unit boundary, and is therefore $\pm 0.3 \mathrm{log}$ unit, due to the coarser grading scale of $0.10 \log$ unit steps. Figure $3 c$ shows a similar plot for traditional Single Letter Acuity. This graph clearly illustrates the dramatic effect of unequal increments in the measuring scale. This test reveals the highest degree of concordance $(61.8 \%)$ indicating a coarse measuring scale (Bailey et al., 1991). A major problem of unequal scale increments is that the confidence intervals vary in magnitude throughout the scale. The amount of change required to exceed the $95 \%$ confidence limits depends critically on the starting point on the scale.

Since confidence limits cannot be established for the Single Letter Acuity test by direct observation of the discrepancy distribution due to the unequal scale increments, an alternative method must be devised. Therefore, the COR was used to calculate the 95\% confidence limit for the Single Letter Acuity test, giving a value of $\pm 0.206 \mathrm{log}$ unit. As measured values must fall outside these limits to constitute a clinically

Table 2. Mean difference, standard deviation and $95 \%$ ranges for the three child test comparisons

\begin{tabular}{|c|c|c|c|c|c|}
\hline & Mean difference & Standard deviation & $95 \%$ range & Rho & Alpha \\
\hline Single Letter Acuity(Log) vs Glasgow Acuity Cards & 0.105 & 0.085 & \pm 0.17 & 0.587 & $<0.01$ \\
\hline Single Letter Acuity vs Glasgow Acuity Cards & 0.149 & 0.094 & \pm 0.18 & 0.536 & $<0.01$ \\
\hline Single Letter Acuity vs Single Letter Acuity(Log) & 0.047 & 0.091 & \pm 0.18 & 0.457 & $<0.01$ \\
\hline
\end{tabular}



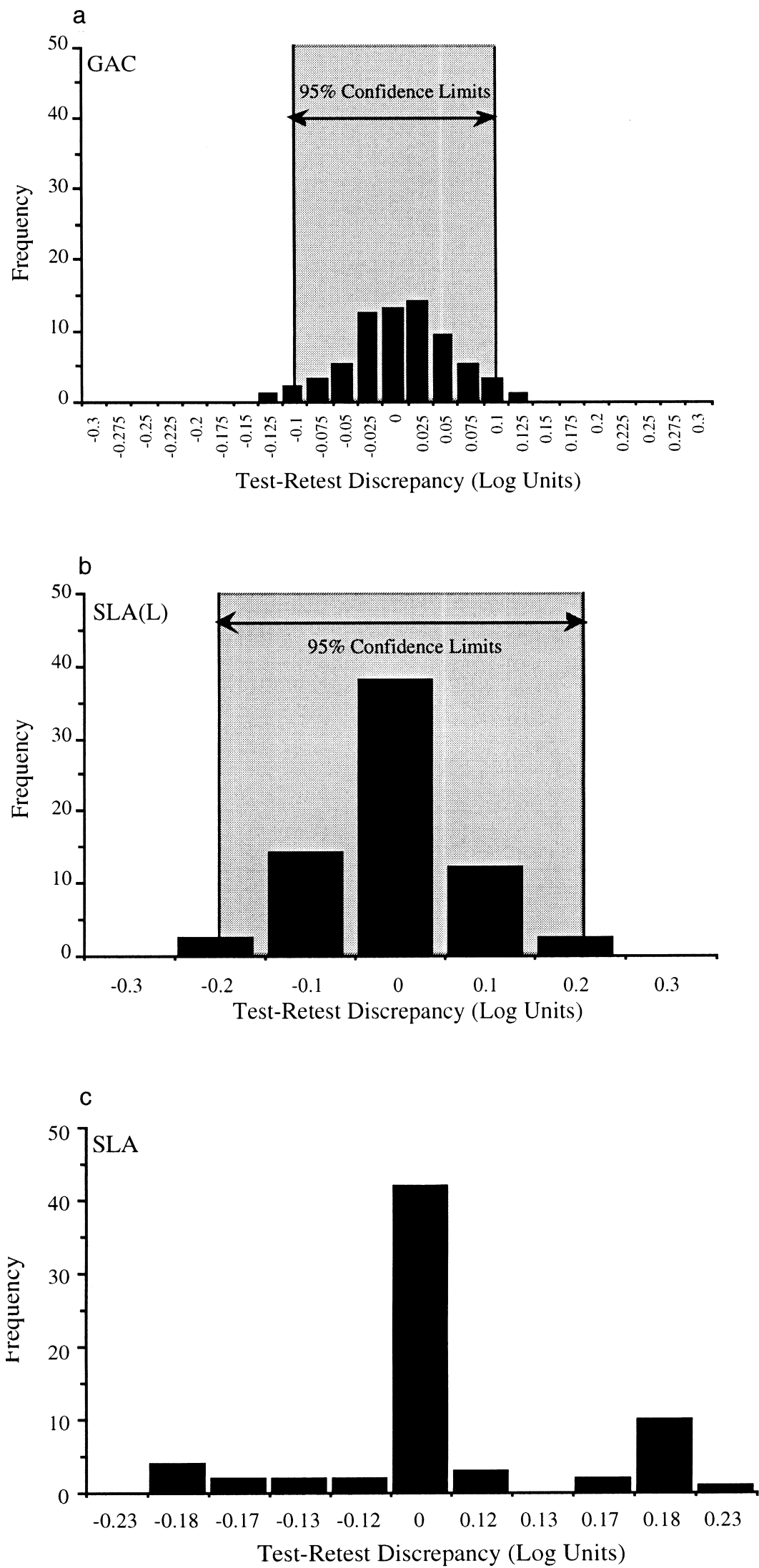

Figure 3. Frequency distribution plots of the discrepancy in acuity score between test and retest sessions (68 visually normal children) for (a): Glasgow Acuity Cards; (b): Single Letter Acuity (Log); (c): Single Letter Acuity. 
significant change, the mean incremental step size (0.144 log unit) has been added. Therefore, in order for the Single Letter Acuity test to detect a real change in visual acuity, a score must change by approximately $\pm 0.35 \log$ unit. The mathematical model described by Bailey et al. (1991) has been used to predict the degree of concordance between test and retest measures, and the $95 \%$ confidence limits for detecting a change in acuity for each test. The results are presented in Table 3.

A key issue in acuity test chart design is the choice of an appropriate grading scale. Consideration of this central feature can improve substantially the clinician's ability to detect or monitor change in a clinical condition, such as amblyopia, using letter acuity measurements. The results of Experiment 2 demonstrate the powerful effect on the confidence limits of increasing the number of sample points on the acuity scale. A clinically significant difference in acuity for Glasgow Acuity Cards is found to be one line and one letter $( \pm 0.125 \log$ unit) as opposed to a three line $( \pm 0.30 \mathrm{log}$ unit) change which is required for Single Letter Acuity ( $\log )$ measures.

Determination of appropriate confidence limits for a Snellen-based chart is extremely difficult as the width of these limits vary depending upon the level of acuity being measured. It was for this reason that a single letter acuity test using a log progression of letter size was introduced into the study design. However, the COR was used to determine an 'approximate' confidence limit for the Single Letter Acuity chart. It should be noted that for optimum use of the COR, the scale should be continuous and the data normally distributed, which is not the case in the Single Letter Acuity test. This suggests that some caution should be exercised when interpreting the confidence limits obtained from the COR.

Tests yielding high degrees of concordance do not necessarily provide narrow confidence limits, which are essential if clinical change is to be detected reliably (Reeves et al., 1987). Indeed, high degrees of concordance indicate that the grading scale is too coarse, needlessly restricting the clinician's ability to detect change (Bailey et al., 1991). Application of the Bailey et al. (1991) mathematical model allows calculation of confidence limits for individual letter scoring to be compared with the more traditional scoring-by-row technique for the Single Letter Acuity ( $\log )$ test. Assigning each letter a score of $0.033 \mathrm{log}$ unit rather than scoring-by-row $(0.10 \log$ unit $)$ the model predicts perfect concordance in $15.7 \%$ of paired comparisons and sets the confidence limits at $\pm 0.192 \log$ unit. Therefore, a significant change in acuity becomes $\pm 0.20 \mathrm{log}$ unit for individual letter scoring as opposed to $\pm 0.30 \log$ unit for scoring by row (the standard de- viation of the discrepancy distribution was derived from the data presented in Figure 3b). Unfortunately, such a comparison between scoring techniques is impractical for the traditional Single Letter Acuity test due to unequal scale increments and the different numbers of optotypes at each acuity level.

It has previously been suggested that to achieve a moderate degree of sensitivity to detecting change in acuity, the magnitude of the scale increment should not be larger than one standard deviation of the testretest discrepancy distribution (Bailey et al., 1991). The results of this study indicate that for the preschool letter acuity charts investigated, only Glasgow Acuity Cards meet this criterion. The Single Letter Acuity ( $\log$ ) test may also meet this criterion if an interpolated scoring by letter procedure were adopted. Experimental data obtained for both Glasgow Acuity Cards and Single Letter Acuity ( $\log$ ) were found to agree well with the model predictions (see Table 3).

\section{Experiment 3. Study of inter-ocular acuity differences in visually normal children and children with amblyopia}

\section{Methods and subjects}

Corrected visual acuity was measured from the right and left eyes of an additional group of 45 visually normal children (mean age $=4.94 \pm 0.55$ years) using Glasgow Acuity Cards, Single Letter Acuity and Single Letter Acuity ( $\log$ ) tests during the same test session. Measurements of acuity were carried out under standard room illumination (500 lux-measured at chart surface, approximately $151 \mathrm{cdm}^{-2}$ ). Tests were again administered in random order and the $95 \%$ confidence limit for a significant difference in acuity between right and left eyes was determined for each test. Acuity measurements were scored using the GAC log scoring system $(1-\log$ MAR).

In addition, corrected right and left eye acuities were also measured from 26 consecutive, clinically diagnosed amblyopic children (mean age $=5.23 \pm 1.21$ years). The diagnosis was made by an ophthalmologist on the basis of Snellen visual acuity measurements and the coexistence of an amblyogenic factor. All amblyopic children attended the Royal Hospital for Sick Children in Glasgow and were either undergoing amblyopia therapy or about to begin therapy. The mean acuity in the amblyopic eyes of this group of children was $0.56 \pm 0.26$ Glasgow Acuity Card log score $(0.44 \log$ MAR, approximately $6 / 15$ or $20 / 50)$.

\section{Results and discussion}

Frequency distribution plots for the right and left 

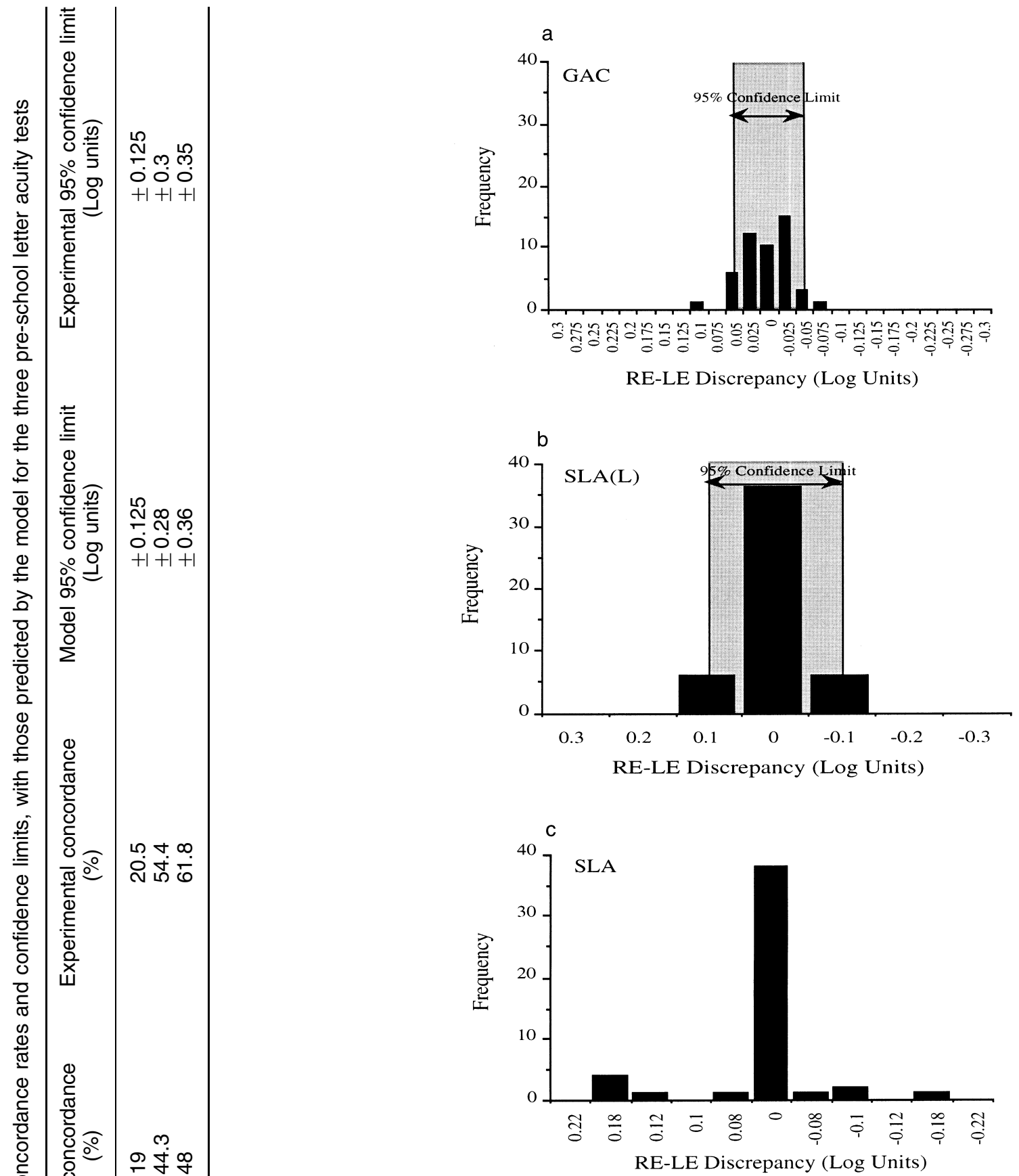

Figure 4. Frequency distribution plots of the discrepancy in acuity score between right and left eyes (45 visually normal children) for (a): Glasgow Acuity Cards; (b): Single Letter Acuity (Log); (c): Single Letter Acuity.

eye discrepancies using Glasgow Acuity Cards, Single Letter Acuity (Log) and Single Letter Acuity are shown in Figures $4 a-c$ respectively. From these data the $95 \%$ confidence limit for each test has been determined and were $\pm 0.05 \mathrm{log}$ unit (Glasgow Acuity Cards) and $\pm 0.10 \log$ unit (Single Letter Acuity (Log)) giving clinically significant difference values of \pm 0.075 
$\log$ unit (Glasgow Acuity Cards) and $\pm 0.20 \log$ unit (Single Letter Acuity (Log)).

The uneven scale increments of the Single Letter Acuity test again confound the assignment of the $95 \%$ confidence limits from direct observation of the frequency distribution plot. The COR is again used to set a value for the mean $95 \%$ confidence limits for a difference in score between eyes. As the 45 children participating in the initial part of Experiment 3 were visually normal and optically corrected, it would be inappropriate to use the mean scale increment for the whole chart, since all the acuity scores will be clustered over a narrow range. Therefore, the mean scale increment was evaluated for the acuity range over which the population scores lay, and was found to be 0.15 $\log$ unit. As the standard deviation of the discrepancy distribution is 0.063 for the Single Letter Acuity test, the COR is $\pm 0.123 \log$ unit. This sets the $95 \%$ confidence limits for a clinically significant difference in acuity score between eyes at approximately $\pm 0.27 \log$ unit.

The $95 \%$ confidence limits for a significant difference in acuity score between right and left eyes of the visually normal cohort, for each of the acuity tests, were used as a criteria for detecting unilateral amblyopia. Any amblyopic subject displaying a difference in acuity between eyes, which exceeded the limits for any of the tests set by data on normal children, was deemed to be successfully detected. The percentage of amblyopic subjects identified correctly by each test were 100\% (Glasgow Acuity Cards), 57.7\% (Single Letter Acuity (Log)) and 42.3\% (Single Letter Acuity).

While detection of changes in acuity longitudinally are important it is also desirable for an acuity test to be able to detect differences in acuity between the eyes of an individual patient. Clinical definitions of unilateral amblyopia commonly require that the visual acuity between the two eyes differs by $2-3$ Snellen lines (Schapero, 1971; Flynn and Cassady, 1978). These figures, although derived from clinical observations and experience, would seem to represent a reasonable criterion as differences of less than three lines are masked by the poor repeatability of the Snellen chart (Gibson and Sanderson, 1980). This criterion is confirmed by the data of the present study for the Snellenbased Single Letter Acuity chart, where a difference between eyes must exceed approximately $\pm 0.27 \mathrm{log}$ unit to reach clinical significance ( three lines). Therefore, the density of amblyopia that can be detected reliably is determined by the sensitivity of the test to detecting differences in acuity between the two eyes. The design features of Glasgow Acuity Cards allow detection of smaller inter-ocular differences in acuity ( $0.075 \log$ unit or $3 / 4$ of a line), improving the tests ability to detect unilateral amblyopia.
Confidence limits for inter-ocular acuity differences were found to be smaller than the confidence limits for test-retest differences for both Glasgow Acuity Cards and the Single Letter Acuity (Log) test. Since corrected visual acuity was measured when defining the confidence limits for inter-ocular acuity, as opposed to uncorrected vision measurements which were made during test-retest sessions (Experiment 2), such a difference is expected. Repeatability of acuity measurements have been shown previously to be greatest when the subject is wearing their optimal refractive correction (Elliott and Sheridan, 1988).

\section{Conclusions}

The results of this study confirm that consideration of important features such as providing an equivalent task at each acuity level, and utilising a logarithmic progression of letter size, in the design of pre-school letter acuity tests, offers substantial improvements in test performance. Sensitivity for detection of change in acuity, and differences in acuity scores between eyes in the same subject, are greater when compared to Single Letter Acuity tests. These features are important for the effective detection and management of children with amblyopia.

\section{Acknowledgements}

This work was partially supported by a grant from the Visual Research Trust. Author PVM is supported by a Vision Research Training Fellowship from the Wellcome Trust.

\section{References}

Atkinson, J., Anker, S., Evans, C., Hall, R. and PimmSmith, E. (1988). Visual acuity testing of young children with the Cambridge Crowding Cards at $3 \mathrm{~m}$ and $6 \mathrm{~m}$. Acta Ophthalmol. 66, 505-508.

Atkinson, J., Pimm-Smith, E., Evans, C., Harding, G., Braddick, O. 1985. Visual crowding in young children. In: Detection and measurement of visual impairment on preverbal children. London: Doc. Ophthalmol. Proc. Ser., 201213.

Bailey, I. L. and Lovie, J. E. (1976). New design principles for visual acuity test charts. Am. J. Optom. Physiol. Opt. 53, 745-753.

Bailey, I., Bullimore, M. A., Raasch, T. and Taylor, H. R. (1991). Clinical grading and the effects of scaling. Invest. Ophthalmol. Vis. Sci. 32, 422-432.

Bennett, A. G. (1965). Ophthalmic test types. Br. J. Physiol. Opt. 22, 238-271.

Bland, J. M. and Altman, D. G. (1986). Statistical methods for assessing agreement between two methods of clinical measurement. Lancet i, 307-310.

Brant, J. C. and Nowotny, M. (1976). Testing of visual 
acuity in young children: an evaluation of some commonly used methods. Develop. Med. Child Neurol. 18, 568-576.

Dreyer, V. (1964). On the exactness of visual acuity determination charts with decimal, Snellen and logarithmic notation. Acta Ophthalmol. 42, 295-306.

Egan, D. F. and Brown, R. (1984). Vision testing of young children in the age range 18 months to 4 years. Child Care Hlth. Dev. 10, 381-390.

Elliott, D. B. and Sheridan, M. (1988). The use of accurate visual acuity measurements in clinical anti-cataract formulation trials. Ophthal. Physiol. Opt. 8, 397-401.

Elliott, D. B., Bullimore, M. A. and Bailey, I. L. (1991). Improving the reliability of the Pelli-Robson contrast sensitivity test. Clin. Vis. Sci. 6, 471-475.

Elliott, D. B., Yang, K. C. H. and Whitaker, D. (1995). Visual acuity changes throughout adulthood in normal, healthy eyes: Seeing beyond 6/6. Optom. Vis. Sci. 72, 186191.

Fern, K. D. and Manny, R. E. (1986). Visual acuity of the pre-school child: a review. Am. J. Optom. Physiol. Opt. 63, 319-345.

Fern, K. D., Manny, R. E., Davis, J. R. and Gibson, R. R. (1986). Contour interaction in the preschool child. Am. J. Optom. Physiol. Opt. 63, 313-318.

Ferris, F. L. and Bailey, I. (1996). Standardising the measurement of visual acuity for clinical research studies - guidelines from the Eye Care Technology Forum. Ophthalmology 103, 181-182.

Ferris, F. L., Kassoff, A., Bresnick, G. H. and Bailey, I. (1982). New visual acuity charts for clinical research. Ophthalmology 94, 91-96.

Flom, M. C., Heath, G. and Takahaski, E. (1963). Contour interaction and visual resolution: contralateral effects. Science 142, 979-980.

Flynn, J. T. and Cassady, J. C. (1978). Current trends in amblyopia therapy. Ophthalmology 85, 428-450.

Gibson, S. A. and Sanderson, H. F. (1980). Observer variation in ophthalmology. Br. J. Ophthalmol. 64, 457-460.

Graham, F. K., Berman, P. W. and Ernhart, C. B. (1960). Development in pre-school children of the ability to copy forms. Child Dev. 31, 339-359.

Hilton, H. F. and Stanley, J. C. (1972). Pitfalls of testing children's vision by the Sheridan Gardiner single optotype method. Br. J. Ophthalmol. 56, 135-139.

Kendall, J., Stayte, M. and Wortham, C. (1989). Ocular defects in children from birth to 6 years of age. $\mathrm{Br}$. Orthopt. J. 46, 3-6.

Kitao, J. (1960). Studies on the development of depth perception for near and distant objects in childhood. Jpn $J$. Ophthalmol. 4, 67-76.

Lovie-Kitchin, J. E. (1988). Validity and reliability of visual acuity measurements. Ophthal. Physiol. Opt. 8, 363-370.

McGraw, P. V. and Winn, B. (1993). Glasgow Acuity Cards: a new test for the measurement of letter acuity in children. Ophthal. Physiol. Opt. 13, 400-404.

Pickert, S. M. and Wachs, H. (1980). Stimulus and communication demands of visual acuity tests. Am. J. Optom. Physiol. Opt. 57, 875-880.

Reeves, B. C., Hill, A. R. and Aspinall, P. A. (1987). The clinical significance of change. Ophthal. Physiol. Opt. 8, 441-446.

Regan, D. (1988). Low contrast letter charts and sine wave grating tests in ophthalmological and neurological disorders. Clin. Vision Sci. 2, 235-250.

Rudel, R. G. and Teuber, H. L. (1963). Discrimination of direction of line in children. J. Comp. Physiol. Psychol. 56, 892-898.

Salt, A. T., Sonksen, P. M., Wade, A. and Jayatunga, R. (1995). The maturation of linear acuity and compliance with the Sonksen-Silver acuity system in young children. Dev. Med. Child Neurol. 37, 505-514.

Schapero, M. (1971). Amblyopia. Chilton, Philadelphia.

Sheridan, M. D. (1960). Vision screening of very young or handicapped children. Br. Med. J. 2, 453-456.

Sheridan, M. D. (1963). Diagnosis of visual defect in early childhood. Br. Orthopt. J. 20, 29-36.

Sheridan, M. D. (1974). What is normal distance vision at five to seven years? Develop. Med. Child Neurol. 16, 189195.

Smorvik, D. and Bosnes, O. (1976). Assessment of visual acuity in pre-school children. Scand. J. Psychol. 17, 122124.

Sokol, S. (1978). Measurement of infant visual acuity from pattern-reversal evoked potentials. Vision Res. 18, 33-39.

Stewart-Brown, S. L., Haslum, M. N. and Howlett, B. (1988). Pre-school vision screening: a service in need of rationalisation. Arch. Dis. Child. 63, 356-359.

Teller, D. Y. (1979). The forced choice preferential looking procedure: a psychophysical technique for use with human infants. Infant Behav. Dev. 2, 135-153.

von Noorden, G. K. (1985). Burian-von Noorden's Binocular Vision and Ocular Motility, 3rd ed. C. V. Mosby, St. Louis.

Westheimer, G. (1979). Scaling of visual acuity measurements. Arch. Ophthalmol. 97, 327-330.

Wick, B. and Schor, C. M. (1984). A comparison of Snellen chart and S-chart visual acuity assessment in amblyopia. $J$. Am. Optom. Assoc. 55, 359-361.

Wild, J. M. and Hussey, M. K. (1985). Some statistical concepts in the analysis of vision and visual acuity. Ophthal. Physiol. Opt. 5, 63-71.

Wohlwill, W. F. (1960). Developmental studies of perception. Psychol. Bull. 57, 249-288.

Youngson, R. M. (1975). Anomaly of visual acuity testing in children. Br. J. Ophthalmol. 59, 168-170. 